\title{
Modelo de Canal PLC para Aplicação em Banda Larga
}

\author{
Bruno L. M. T. Silva, Jefferson C. Silva e Suzete E. N. Correia
}

\begin{abstract}
Resumo-O presente trabalho tem como objetivo apresentar uma implementação de um canal PLC (Power Line Communication), que recentemente tem sido usado para a transmissão de sinais em banda larga . Os resultados obtidos atestam que este canal é seletivo em freqüiência e possui severas atenuações com o aumento da freqüência e comprimento do canal.
\end{abstract}

Palavras-Chave-Transmissão em banda larga, Canais PLC, Multipercurso, Seletividade em freqüência.

Abstract-The objective of this work is to present an implementation of the PLC channel, which has been recently used for the transmission of broadband signals. Experimental results show that this channel is selective in frequency and has severe attenuations with the increase of the frequency and the channel length.

Keywords-Broadband transmission, PLC channel, Multipath, Frequency selective.

\section{INTRODUÇÃO}

A crescente demanda por acesso rápido à internet e por novos serviços tem incentivado o estudo de diversos tipos de meios de transmissão de dados, principalmente, em banda larga. Recentemente, o uso das redes elétricas como meio de transmissão de dados, conceituadas de canais PLC (Power Line Communication), têm recebido atenção por apresentarem uma largura de banda de $30 \mathrm{MHz}$ e capacidade de transmissão de dados acima de algumas centenas de Mbit/s. Além do mais, estão presentes em cerca de $95 \%$ dos domicílios residenciais, comerciais e industriais e apresentam baixo custo de implementação [1], [2].

As redes elétricas diferem consideravelmente em termos de estrutura, topologia, e propriedades físicas dos convencionais meios de transmissão guiados, tais como fio telefônico, par trançado e cabo coaxial. Dentre as diferenças pode-se citar o compartilhamento do mesmo meio físico de comunicação por vários usuários e a presença de várias fontes de ruído [1], [2]. Além disso, os canais PLC podem ser vistos como um misto entre um canal de telefonia fixa e um canal de comunicação sem fio, uma vez que os mesmos apresentam desvanecimentos seletivos em frequiência causados pela propagação em múltiplos percursos e severas atenuações em função do comprimento do canal, além do ruído impulsivo de natureza não gaussiana com potência suficiente para corromper rajadas de bits [1].

Os canais PLC estão dispostos em dois ambientes possíveis: os last miles ou outdoor que compreendem a rede elétrica externa de média e alta tensão e os ambientes last meters ou

Coordenação de Telecomunicações, Centro Federal de Educação Tecnológica da Paraíba, João Pessoa, Brasil, E-mail: brunleo_22@oi.com.br, jefferson@cefetpb.edu.br, suzete@cefetpb.edu.br. indoor que compreendem a rede elétrica de baixa tensão e apresentam características mais favoráveis do que os canais outdoor, pelo fato de não estarem sujeitos a fatores externos como a interferência por ondas curtas de rádio [1]. Sob o ponto de vista de transmissão em banda larga, a faixa de freqüências de $500 \mathrm{KHz}$ a $10 \mathrm{MHz}$ é destinada para canais outdoor e faixa de freqüências de $10 \mathrm{MHz}$ a $30 \mathrm{MHz}$ para canais indoor [1].

Neste trabalho, é apresentando uma implementação computacional do canal PLC, considerado-se o ambiente last meters na largura de banda de $20 \mathrm{MHz}$. Na seção II, o modelo do canal empregado na simulação é descrito. A seção III traz os resultados obtidos para as condições de canal empregadas e, por fim, na seção IV são apresentadas as conclusões e perspectivas futuras do trabalho.

\section{Modelo do CANAL PLC}

O estudo do canal PLC pode ser baseado em dois métodos: o button up que descreve o canal segundo matrizes de admitância e impedância, necessitando de um conhecimento apurado da rede e o top down que descreve o canal segundo uma função de transferência que corresponde a resposta em frequiência do canal, possibilitando uma representação simples e independente da topologia da rede elétrica. Dentre os modelos de canais PLC que são descritos através de uma função de transferência, o modelo proposto por Zimmerman e Dosdert [3] para ambientes last miles pode ser empregado para ambientes last meters sem perda de generalidade. Tal modelo é o mais atual e utilizado por levar em consideração diversos parâmetros, tais como fatores de transmissão e reflexão de ondas, atenuação em função do comprimento do canal e os multipercursos e seus respectivos atrasos. A equação 1 mostra a função de transferência complexa de um típico canal PLC:

$$
\begin{gathered}
H(f)=\sum_{i=0}^{n} G(i) e^{\left(-a_{0}+a_{1} f^{k} d(i)\right)} e^{-j 2 \pi \tau(i)}, \\
\tau(i)=\frac{d(i) \sqrt{\varepsilon_{r}}}{c_{0}}
\end{gathered}
$$

em que $i$ representa o número de percursos, $a_{0}$ e $a_{1}$ são os parâmetros de atenuação, $k$ é o expoente do fator de atenuação, $d(i)$ é o comprimento do canal no i-ésimo percurso e $\tau(i)$ é o atraso devido ao multipercurso, o qual depende da velocidade da luz $c_{0}$ e da constante dielétrica do cabo $\varepsilon_{r}$, como mostra a equação 2. A função $G(i)$ é um fator de ponderação obtido pelo produto dos fatores de transmissão e reflexão nos vários percursos. O módulo de $G(i)$ no percurso $i$ tem um limitante superior dado por [1], [3]:

$$
|G(i)| \leq 1 .
$$


A equação 1 pode ser usada para modelar canais PLC na faixa de freqüências de $500 \mathrm{KHz}$ a $20 \mathrm{MHz}$ [3].

\section{SimulaçÕES}

Nas simulações, foram considerados um expoente do fator de atenuação $K=1$, parâmetros de atenuação $a_{0}=$ $-0,02.10^{-3}$ e $a_{1}=8,1.10^{-10}$ e seis percursos cujos comprimento $d(i)$ e fatores de ponderação $G(i)$ encontram-se na Tabela I. Tais dados são fornecidos por [2], [3]. A faixa de freqüência considerada foi de 0 a $20 \mathrm{MHz}$.

TABELA I

PARÂMETROS DO CANAL

\begin{tabular}{|c|c|c|c|c|c|c|}
\hline Percurso & 1 & 2 & 3 & 4 & 5 & 6 \\
\hline Comprimento $(\mathrm{m})$ & 200 & 221 & 242 & 259 & 266 & 530 \\
\hline Fator de \\
Ponderação & 0,54 & 0,275 & $-0,15$ & 0,08 & $-0,03$ & $-0,02$ \\
\hline
\end{tabular}

A densidade espectral de potência do canal PLC para a faixa de $20 \mathrm{MHz}$ encontra-se ilustrada na Figura 1. Em virtude da variação de potência em função da frequiência, observase que o canal é seletivo em freqüência, ocorrendo uma forte atenuação do sinal em função da faixa de freqüência utilizada. Tais características se mantêm quando se aumenta o comprimento do canal para valores acima de algumas centenas de metros, comprimentos estes ainda admissíveis para ambientes last meters. Entretanto, como mostra a Figura 2 a curva adquire um comportamento quase linear e a atenuação aumenta.

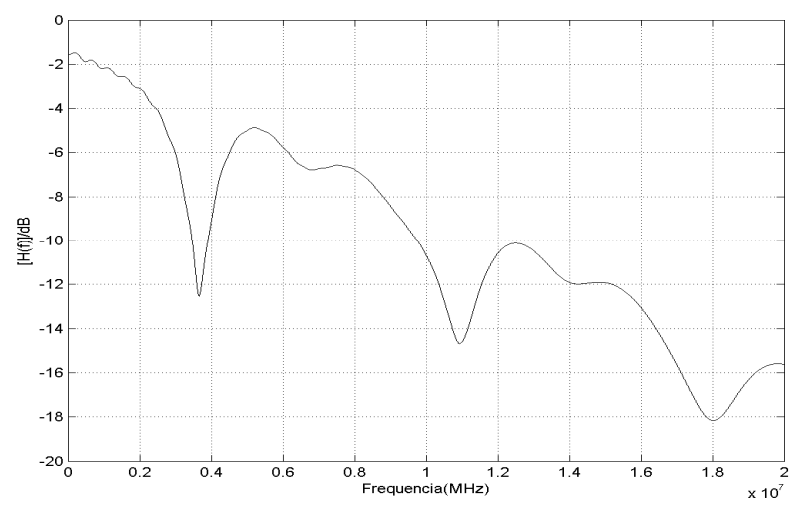

Fig. 1. Densidade espectral de potência do canal PLC.

A resposta em fase do canal é ilustrada na Figura 3. As simulações mostram que a linearidade da fase do canal PLC implica que o mesmo apresenta um deslocamento de fase constante, podendo ser estimado com uso de portadoras pilotos [4]. Para o desvanecimento seletivo em freqüência presente neste canal, que demanda do sistema complexos esquemas de equalização temporal, atribui-se o uso de técnicas de multiportadoras, a exemplo da técnica OFDM (Orthonal Frequency Division Multiplexing) [1], [2], [4] que combate tal desvanecimento, tornando o processo de equalização simples ou desnecessário, além de eliminar a interferência intersimbolica e permitir o uso eficiente da largura de banda do canal.

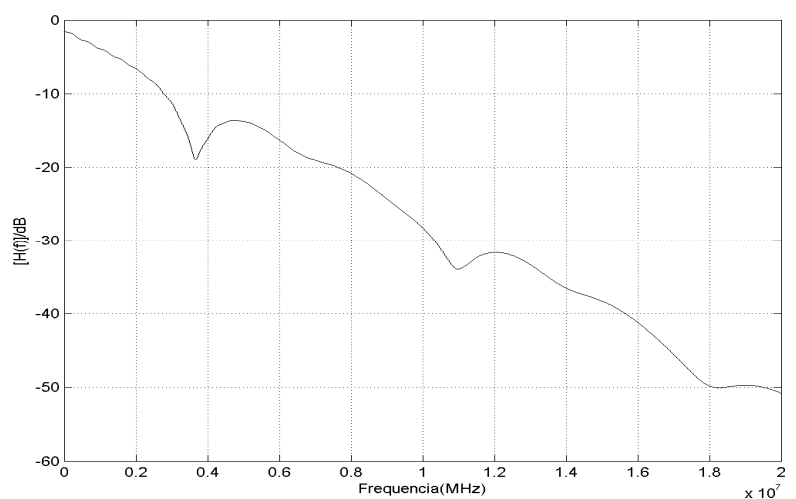

Fig. 2. Densidade espectral de potência do canal PLC considerando o aumento do comprimento do canal.

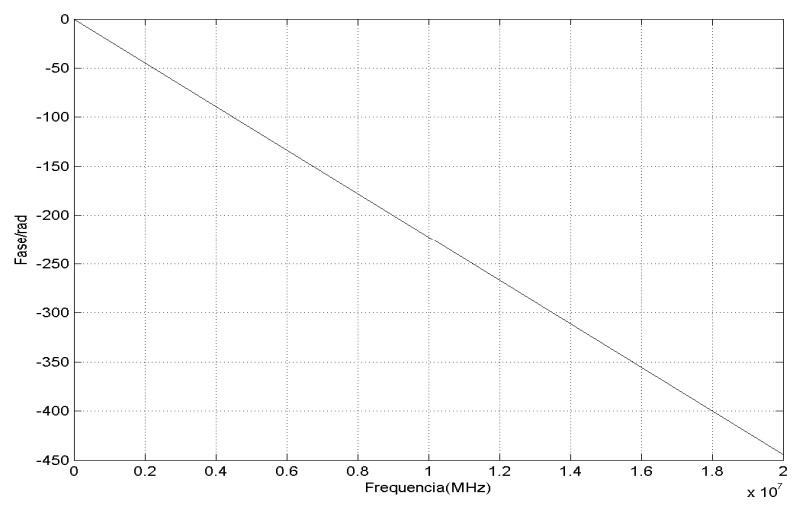

Fig. 3. Resposta em fase do canal.

\section{CONCLusões}

Neste trabalho foi implementado um modelo de canal PLC considerado-se o ambiente last meters na largura de banda de $20 \mathrm{MHz}$. Os resultados obtidos comprovam que os canais PLC apresentam uma forte atenuação em função dos múltiplos percursos e do comprimento do canal, bem como seletividade em freqüência. $\mathrm{O}$ modelo implementado não leva em consideração o ruído característico da própria rede elétrica, como também os fatores externos decorrentes do chaveamento, transitórios e injeção de cargas de teor reativo. Em trabalho futuros, pretende-se desenvolver soluções que considerem a adição do ruído e que permitam não só analise do canal como processamento de sinais segundo estas características.

\section{REFERÊNCIAS}

[1] V. Ribeiro, "Técnicas de Processamento de Sinais Aplicadas à Transmissão de Dados via Rede Elétrica e Monitoramento da Qualidade de Energia", Tese de Doutorado, Faculdade de Engenharia Elétrica e de Computação, Universidade Estadual de Campinas, 2005.

[2] J. Silva et. al., "Técnica OFDM Aplicada a Power Line Communications, Congresso de Inovação Tecnológica em Energia Elétrica, 2003.

[3] M. Zimmemann e K. Dosdert, "A Multi-Path Signal Propagation Model for the Power Line Channel in the High Frequency Range, Proceedings of the 3rd. International Symposium on Power Line, p. 45-51, 1999.

[4] E. Pinto e C. Albuquerque, "A Técnica de Transmissão OFDM", Revista Científica Periódica - Telecomunicações, v. 5, n.1, Junho 2002. 